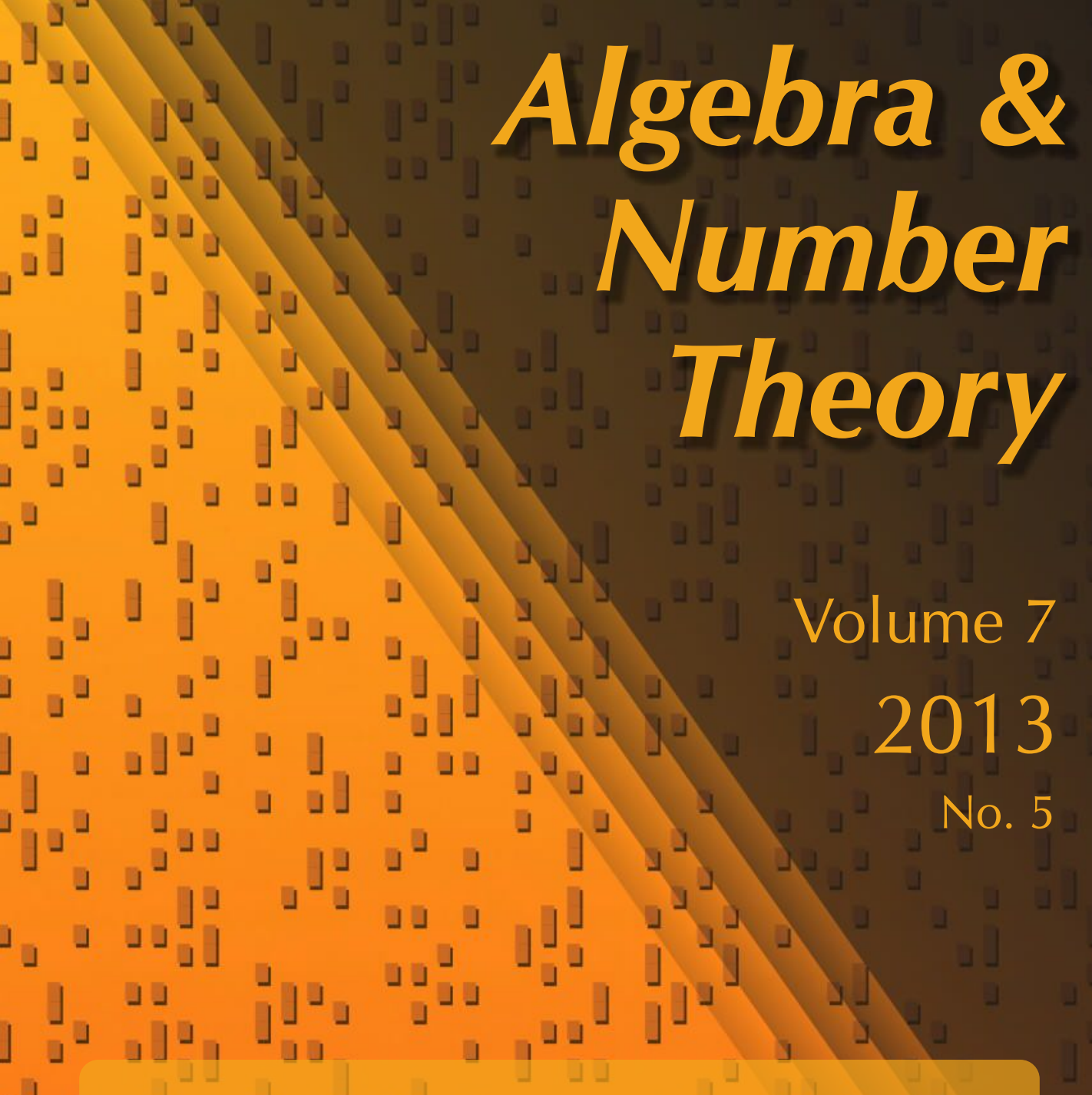




\title{
On binary cyclotomic polynomials
}

\author{
Étienne Fouvry
}

We study the number of nonzero coefficients of cyclotomic polynomials $\Phi_{m}$, where $m$ is the product of two distinct primes.

\section{Presentation of the results}

Let $m \geq 1$ be an integer, and let $\Phi_{m}$ be the cyclotomic polynomial defined by

$$
\Phi_{m}(X):=\prod_{\substack{j=1 \\(j, m)=1}}^{m}(X-\exp (2 \pi i j / m)) .
$$

This monic polynomial belongs to $\mathbb{Z}[X]$, and its degree is equal to $\varphi(m)$, the Euler function of the integer $m$. Let $\theta(m)$ be the number of nonzero coefficients of $\Phi_{m}$. Of course, $\theta(m)$ satisfies the trivial inequalities

$$
2 \leq \theta(m) \leq \varphi(m)+1,
$$

which are optimal when one considers the case $m=1$ or $m=p$, a prime number. In these cases, all of the coefficients of $\Phi_{m}$ are equal to 1 .

We reserve the letters $p$ and $q$ for prime numbers. We call an integer $m$ binary if it is of the form $m=p q$, with $p$ and $q$ distinct. Let $\mathscr{B}=\{6,10,14,15,21, \ldots\}$ be the set of binary integers. For $m \in \mathscr{B}$, we say that the associated cyclotomic polynomial $\Phi_{m}$ is binary. The coefficients of the binary cyclotomic polynomial $\Phi_{m}$ are equal to 0,1 or -1 . Furthermore, in that particular case, the function $\theta(m)$ has an explicit expression in terms of $p$ and $q$ that can be exploited by analytic number theory. More precisely:

Proposition A. Let $m=p q$ be a binary integer with $p \neq q$. Then we have

$$
\theta(m)=2 \bar{p}_{q} \bar{q}_{p}-1,
$$

where $\bar{p}_{q}$ is the unique integer satisfying

$$
\bar{p}_{q} p \equiv 1 \bmod q \text { and } 1 \leq \bar{p}_{q}<q
$$

and $\bar{q}_{p}$ is defined similarly.

MSC2010: primary 11N13; secondary 11L20, 11C08, 11N36, 11L07, 11N05.

Keywords: cyclotomic polynomials, exponential sums, sieves. 
For a proof of this basic result, see [Carlitz 1966, Theorem; Bzdęga 2012], and for an interesting characterization of the nonzero coefficients of $\Phi_{p q}$, see [Lam and Leung 1996] for instance.

Recently Bzdęga [2012] started the study of the distribution function of the map

$$
m \in \mathscr{B} \mapsto \theta(m) .
$$

Let us review his results. Let $\gamma$ and $x$ be real numbers satisfying $0<\gamma<\frac{1}{2}$ and $x \geq 6$, and let $H_{\gamma}(x)$ be the counting function

$$
H_{\gamma}(x):=\#\left\{m: m \in \mathscr{B}, m \leq x, \theta(m) \leq m^{\frac{1}{2}+\gamma}\right\}
$$

(because of the inequality (12) below, it is useless to study $H_{\gamma}$ for $\gamma \leq 0$ ). With these conventions, Bzdęga [2012, Theorem] proved the following:

Theorem A. For every $0<\gamma<\frac{1}{2}$ and every $\epsilon>0$, there exist $C(\gamma), c(\epsilon, \gamma)>0$ and $x_{0}=x_{0}(\epsilon, \gamma)$ such that for $x \geq x_{0}$ one has the inequalities

$$
c(\epsilon, \gamma) x^{\frac{1}{2}+\gamma-\epsilon} \leq H_{\gamma}(x) \leq C(\gamma) x^{\frac{1}{2}+\gamma} .
$$

The idea of Bzdęga is to relate the integers $m=p q$ contributing to $H_{\gamma}(x)$ to the solutions of the equations

$$
\ell q-n p=1,
$$

where $\ell$ and $n$ are integers satisfying some inequalities depending on $p, q$ and $\gamma$. Write $t=n p$. By (4) and by ingenious considerations, he is led to counting integers $t$ such that $t$ and $t+1$ both have a large prime factor. Appealing to a deep result of Hildebrand [1985] on $p$-stable subsets of integers, Bzdegga deduces the inequalities (3).

Our plan is to study (4) in the context of prime number theory and to get three different types of results according to the size of $\gamma$. These results suggest that this investigation becomes more and more intricate as $\gamma$ decreases to 0 . The first result gives an asymptotic formula when $\gamma$ is large. Its proof is mainly based on bounds for Kloosterman-Ramanujan sums over primes (see Lemmas 2 and 3 below) and on the Bombieri-Vinogradov theorem (see Lemma 5).

Theorem 1. For $0<\gamma<\frac{1}{2}$, let

$$
C(\gamma):=\frac{2}{1+2 \gamma} \log \frac{1+2 \gamma}{1-2 \gamma} .
$$

Then for every $\gamma_{0}>0$, uniformly for $\gamma$ satisfying $\frac{12}{25}+\gamma_{0} \leq \gamma \leq \frac{1}{2}-\gamma_{0}$, we have

$$
H_{\gamma}(x) \sim C(\gamma) \frac{x^{\frac{1}{2}+\gamma}}{\log x}
$$

as $x \rightarrow \infty$. 
The second result produces a universal upper bound for $H_{\gamma}(x)$ and is a rather direct consequence of the two-dimensional sieve (see Lemma 4).

Theorem 2. For every $\gamma_{0}>0$, there exists $C^{+}\left(\gamma_{0}\right)$ such that, for every $\gamma$ satisfying $\gamma_{0} \leq \gamma \leq \frac{1}{2}-\gamma_{0}$ and for every $x \geq 6$, the following inequality holds:

$$
H_{\gamma}(x) \leq C^{+}\left(\gamma_{0}\right) \frac{x^{\frac{1}{2}+\gamma}}{\log x} .
$$

The last result is a lower bound when $\gamma$ is large enough. Judging by the tools involved, it is certainly the deepest of our three results (see Lemma 7).

Theorem 3. For every $\gamma_{0}>0$, there exist $C^{-}\left(\gamma_{0}\right)>0$ and $x\left(\gamma_{0}\right)$ such that, for every $\gamma$ satisfying $\frac{15}{98}+\gamma_{0} \leq \gamma \leq \frac{1}{2}-\gamma_{0}$ and for every $x \geq x\left(\gamma_{0}\right)$, the following inequality holds:

$$
H_{\gamma}(x) \geq C^{-}\left(\gamma_{0}\right) \frac{x^{\frac{1}{2}+\gamma}}{\log x} .
$$

When $\gamma=\frac{1}{2}, H_{\gamma}(x)$ counts the number of binary integers less than $x$, and this number is asymptotic to $x(\log \log x)(\log x)^{-1}$. This explains why the asymptotic formula in Theorem 1 cannot be uniform for $\gamma<\frac{1}{2}$. Finally, we postpone to Section 7 a discussion on a conjectural value of $H_{\gamma}(x)$.

\section{Tools}

\subsection{Notation.}

- We reserve the letters $p$ and $q$ for distinct prime numbers. For brevity, we replace the symbols $\bar{p}_{q}$ and $\bar{q}_{p}$ (defined in Proposition A) by $\bar{p}$ and $\bar{q}$.

- For $x \geq 1, \mathscr{L}$ denotes $\log 2 x$, and $\xi:=1+\mathscr{L}^{-1}$.

- For $N \geq 1$, the notation $n \sim N$ and $n \approx N$ respectively replaces the conditions $N<n \leq 2 N$ and $N<n \leq \xi N$.

- For $N \geq 1$, the notation $n \asymp N$ means that $n$ satisfies $c_{1} N<n \leq c_{2} N$, where $0<c_{1}<c_{2}$ are absolute constants that are useless to specify.

- For $x \geq 1, \pi(x)$ is the number of primes less than $x$.

- For integers $r$ and $s, \pi(x ; r, s)$ is the number of $p$ s less than $x$ and congruent to $s$ modulo $r$.

- For a real number $t, e(t)$ is the additive character $\exp (2 \pi i t)$.

- The number of positive divisors of the integer $n$ is denoted by $\tau(n)$. 
2.2. Trigonometric sums. To detect the oscillations of the fractional part of the quotient $\bar{q} / p$, we shall appeal to the following well known lemma of Vinogradov, which is stated in different ways in the literature:

Lemma 1 [Vinogradov 1954, Lemma 12, page 32]. Let $r \geq 1$ be an integer, and let $\beta$ and $\Delta$ be real numbers satisfying $0<\Delta<\beta / 2<1 / 4$. Then there exist two functions $\psi^{ \pm}$with period 1 satisfying

$$
\begin{aligned}
& \begin{cases}\psi^{+}(t)=1 & \text { for } 0 \leq t \leq \beta, \\
0 \leq \psi^{+}(t) \leq 1 & \text { for }-\Delta \leq t \leq 0 \text { or } \beta \leq t \leq \beta+\Delta, \\
\psi^{+}(t)=0, & \text { if } t(\bmod 1) \notin[-\Delta, \beta+\Delta],\end{cases} \\
& \begin{cases}\psi^{-}(t)=1 & \text { for } \Delta \leq t \leq \beta-\Delta, \\
0 \leq \psi^{-}(t) \leq 1 & \text { for } 0 \leq t \leq \Delta \text { or } \beta-\Delta \leq t \leq \beta, \\
\psi^{-}(t)=0, & \text { if } t(\bmod 1) \notin[0, \beta],\end{cases}
\end{aligned}
$$

and

$$
\psi^{ \pm}(t)=\sum_{m=-\infty}^{\infty} c_{m}^{ \pm} e(m t) \text { for every real } t
$$

The coefficients $c_{m}^{ \pm}$satisfy the equalities $c_{0}^{ \pm}=\beta \pm \Delta$ and the inequalities

$$
\left|c_{m}^{ \pm}\right| \leq 2 \min \left\{\beta \pm \Delta, \frac{1}{\pi|m|}, \frac{1}{\pi|m|}\left(\frac{r}{\pi|m| \Delta}\right)^{r}\right\}, \quad m \neq 0 .
$$

2.3. Kloosterman-Ramanujan sums over primes. For real $y \geq x \geq 1$ and for $a$ a nonzero integer, we introduce the following trigonometric sum over primes:

$$
S_{p}(a ; x, y):=\sum_{x<q<y} e\left(a \frac{\bar{q}}{p}\right) .
$$

This sum differs from a classical Kloosterman-Ramanujan sum by the fact that the summation is restricted to prime values. We will benefit from oscillations of the function $q \mapsto e(a(\bar{q} / p))$ under the form of the two following lemmas extracted from [Fouvry and Shparlinski 2011]. The proofs of these two lemmas are based on the method of Garaev [2010]. For more general results on sums of this type, see [Fouvry and Michel 1998].

The first of these two lemmas considers the case where $p$ is small compared with $x$ and $y$.

Lemma 2 [Fouvry and Shparlinski 2011, Theorem 3.2]. The bound

$$
S_{p}(a ; x, y) \ll p^{-\frac{1}{2}} x \mathscr{L}^{2}+p^{\frac{1}{4}} x^{\frac{4}{5}} \mathscr{L}^{\frac{3}{2}}
$$

holds uniformly for every prime $p \geq 2$, for every integer a not divisible by $p$ and for every $1 \leq x \leq y \leq 2 x$. 
This bound is interesting for $p \leq x^{\frac{4}{5}}$ only. We will have to deal with sums $S_{p}(a ; x, y)$ for $p$ slightly less than $x$. Still based on the method of Garaev, we have the following average bound of this sum, which is Theorem 3.3 of [Fouvry and Shparlinski 2011] for the choices $x_{p}=x$ and $x_{p}^{\prime}=2 x$; the extension to the statement given is straightforward.

Lemma 3. For every $\epsilon>0$, the inequality

$$
\sum_{p \sim P} \max _{(a, p)=1}\left|S_{p}\left(a ; x_{p}, x_{p}^{\prime}\right)\right| \ll_{\epsilon}\left(x^{\frac{3}{5}} P^{\frac{13}{10}}+x^{\frac{5}{6}} P^{\frac{13}{12}}\right) P^{\epsilon}
$$

holds uniformly for $P^{\frac{3}{2}} \geq x \geq 1$ and for any sequences of integers $\left(x_{p}\right)_{p \sim P}$ and $\left(x_{p}^{\prime}\right)_{p \sim P}$ satisfying $x \leq x_{p} \leq x_{p}^{\prime} \leq 2 x$.

2.4. The two-dimensional sieve. The following lemma can be obtained by Brun's sieve and will be used in the proof of Theorem 2 since it produces an upper bound for the number of solutions to (4) with a large uniformity over $\ell$ and $n$ :

Lemma 4 [Friedlander and Iwaniec 2010, Proposition 6.22]. Let $a, b$ and $h$ be positive integers satisfying

$$
(a, b)=(a b, h)=1 \quad \text { and } 2 \mid a b h .
$$

Let $N_{a b h}(x, z)$ be the number of pairs of positive integers $m$ and $n$ satisfying am $\leq x$, $(m n, h)=1, a m+h=b n$ and $m n$ has no prime factors less than $z$. Then, for $z \geq 2$ and

$$
x \geq \tau(h) a b z(\log z)^{4},
$$

we have the inequality

$$
N_{a b h}(x, z) \ll \frac{h x}{\varphi(a b h)}(\log z)^{-2},
$$

where the implied constant is absolute.

2.5. The Bombieri-Vinogradov theorem. We now recall this cornerstone of current analytic number theory. It gives the average behavior of the function $\pi(x ; r, s)$ and replaces the assumption of the Generalized Riemann Hypothesis for Dirichlet $L$-functions in many applications. Among the numerous possible references, we give here the version in [Iwaniec and Kowalski 2004, Theorem 17.1, (17.24)].

Lemma 5. For every $A \geq 0$, there exists $C(A)$ such that, for every $x \geq 1$ and for

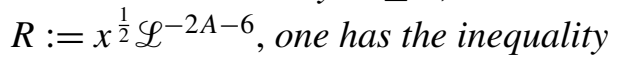

$$
\sum_{r \leq R} \max _{(s, r)=1}\left|\pi(x ; r, s)-\frac{\pi(x)}{\varphi(r)}\right| \leq C(A) x \mathscr{L}^{-A-1} .
$$


2.6. A variant of the Brun-Titchmarsh theorem. The proof of Theorem 3 heavily depends on lower bounds for the function $\pi(x ; r, s)$ in cases that are not covered by Lemma 5, which means $r$ is larger than $x^{\frac{1}{2}}$. We first recall the original statement of Mikawa [2001, Theorem].

Lemma 6. Let $L>\frac{32}{17}$ and $A, B>0$ be given. Let $s$ be an integer and $R$ be large with $0<|s| \leq(\log R)^{B}$. Then, except possibly for $O\left(R(\log R)^{-A}\right)$ integers $r$ satisfying $(r, s)=1$ and $r \sim R$, we have

$$
\inf \{p: p \equiv s \bmod r\} \ll r^{L},
$$

where the implied constants depend only on $A, B$ and $L$.

This result can be interpreted as an average version of Linnik's famous theorem concerning the least prime in an arithmetic progression. Actually, Mikawa's proof gives more. For instance, it instantly gives a lower bound with the correct order of magnitude for the function $\pi\left(r^{L} ; r, s\right)$ for almost all $r$ as above. Due to the value of $L$, this result can be viewed as a lower bound of the function $\pi(x ; r, s)$ for almost all $r$ coprime with $s$ and slightly larger than $\sqrt{x}$. As far as we know, the first result of that type was due to Rousselet [1988] following techniques of Fouvry [1985], who was dealing with upper bounds of the function $\pi(x ; r, s)$ (Brun-Titchmarsh theorem on average). The problem of giving both upper and lower bounds for $\pi(x ; r, s)$ for almost $r$ in the interval $\left[x^{\frac{1}{2}}, x^{\frac{1}{2}+\delta}\right]$, where $\delta$ is a small positive constant, was then treated in several remarkable papers [Bombieri et al. 1987; 1989; Baker and Harman 1996].

We give an improved version of Lemma 6 where we count primes in the interval ]$x, 2 x]$ with some uniformity over the congruence class $\bar{s} \bmod r$ (as above, $\bar{s}$ is the multiplicative inverse of $s \bmod r$ ). Such a generalization is necessary for our application and is possible by the structure of the proof of Lemma 6 based on bounds for Kloosterman sums on average (see [Habsieger and Sivak-Fischler 2010, Theorem 1.5] for another reference where this extension is made).

Lemma 7. For every $K<\frac{17}{32}$, there exist $\alpha_{K}>0, \beta_{K}>0$ and $x_{K}$ such that for every $x>x_{K}$, every $R$ satisfying $2 \leq R<x^{K}$ and every s such that $1 \leq|s| \leq x^{\beta_{K}}$, the inequality

$$
\pi(2 x ; r, \bar{s})-\pi(x ; r, \bar{s}) \geq \alpha_{K} \frac{x}{\varphi(r) \log x},
$$

holds for every $r \sim R$ coprime with $s$ with at most $R(\log R)^{-2}$ exceptions.

Remark. Of course, in this lemma, we can suppose that the functions $K \mapsto \alpha_{K}$ and $K \mapsto \beta_{K}$ are decreasing and $K \mapsto x_{K}$ is increasing. 


\section{Basic transformations}

3.1. Properties of the function $\boldsymbol{\theta}$. We first write the expression of $\theta(p q)$ given by Proposition A in an asymmetrical way. Actually, Bézout's identity and the inequalities $1 \leq \bar{p}<q$ and $1 \leq \bar{q}<p$ lead to the equality

$$
p \bar{p}+q \bar{q}=1+p q,
$$

which transforms (1) into

$$
\theta(p q)=2 p q \cdot \frac{\bar{q}}{p}\left(1+\frac{1}{p q}-\frac{\bar{q}}{p}\right)-1 .
$$

Now suppose that $p<q$. From the trivial inequalities

$$
\frac{1}{p} \leq \frac{\bar{q}}{p} \leq 1-\frac{1}{p}
$$

and from the properties of the function $t \mapsto t((1+1 / p q)-t)$, we deduce

$$
\theta(p q) \geq q>(p q)^{\frac{1}{2}},
$$

which implies that $H_{\gamma}(x)=0$ for $\gamma \leq 0$.

We now want to translate in an efficient manner the inequality

$$
\theta(p q) \leq(p q)^{\frac{1}{2}+\gamma} .
$$

In order to control uniformity aspects, we will frequently assume that we have

$$
\gamma_{0} \leq \gamma \leq \frac{1}{2}-\gamma_{0}
$$

where $\gamma_{0}$ is a fixed positive number.

For $t \geq T\left(\gamma_{0}\right)$, let $0<\theta_{0}(t)<1-\theta_{1}(t)<1$ be the solutions of the polynomial equation of degree 2 in the unknown $X$

$$
2 t X\left(1+\frac{1}{t}-X\right)-1=t^{\frac{1}{2}+\gamma}
$$

For simplicity, we omit in the sequel the dependency on the parameter $\gamma$.

Lemma 8. We suppose that (13) holds. Let $m=p q$ be a binary integer with $p<q$ and $m \geq T\left(\gamma_{0}\right)$. Then

$$
\theta(m) \leq m^{\frac{1}{2}+\gamma} \Longleftrightarrow 0<\frac{\bar{q}}{p} \leq \theta_{0}(m) \text { or } 1-\theta_{1}(m) \leq \frac{\bar{q}}{p}<1 .
$$

The functions $t \mapsto \theta_{0}(t), \theta_{1}(t)$ are decreasing for $t>T\left(\gamma_{0}\right)$, are of $\mathscr{C}^{\infty}$-class and satisfy

$$
\theta_{0}(t), \theta_{1}(t)=\frac{t^{\gamma-\frac{1}{2}}}{2}+O\left(t^{2 \gamma-1}\right),
$$

where the implied constant depends on $\gamma_{0}$ only. 
Proof. The proof of (14) is easy; it is only a transcription of (11). Finally, the asymptotic behaviors of the functions $\theta_{i}(t)$ are consequences of the exact formula

$$
\theta_{0}(t), 1-\theta_{1}(t)=\frac{1+\frac{1}{t} \mp \sqrt{\left(1+\frac{1}{t}\right)^{2}-2 \frac{t^{1 / 2+\gamma}+1}{t}}}{2} .
$$

3.2. Decomposition of $\boldsymbol{H}_{\gamma}(\boldsymbol{x})$. We always suppose that (13) is true. Let $T\left(\gamma_{0}\right)$ be defined as in Lemma 8. We use (14) to split the set contributing to $H_{\gamma}(x)$

$$
\left\{(p, q): p<q, T\left(\gamma_{0}\right) \leq p q \leq x, \theta(p q) \leq(p q)^{\frac{1}{2}+\gamma}\right\}
$$

into two disjoint subsets corresponding to $0<\bar{q} / p \leq \theta_{0}(p q)$ or $1-\theta_{1}(p q) \leq \bar{q} / p<1$. Let $H_{\gamma}^{0}(x)$ and $H_{\gamma}^{1}(x)$ be the corresponding cardinalities, which give the equality

$$
H_{\gamma}(x)=H_{\gamma}^{0}(x)+H_{\gamma}^{1}(x)+O\left(T\left(\gamma_{0}\right)\right) .
$$

We shall concentrate our study on the case of $H_{\gamma}^{0}(x)$ since the case of $H_{\gamma}^{1}(x)$ is quite similar because the functions $\theta_{0}$ and $\theta_{1}$ play the same role (see Lemma 8).

To control the order of magnitude of the variables $p$ and $q$, we consider, for $P, Q \geq 2$ such that $P Q \geq T\left(\gamma_{0}\right)$, the counting functions

$$
R_{\gamma}(P, Q):=\#\left\{(p, q): p<q, p q \leq x, p \approx P, q \approx Q, 0<\frac{\bar{q}}{p} \leq \Theta_{0}\right\},
$$

where

$$
\Theta_{0}=\theta_{0}(P Q) .
$$

Since the function $\theta_{0}$ is decreasing, we obtain the inequality

$$
H_{\gamma}^{0}(x) \leq \sum_{P} \sum_{Q} R_{\gamma}(P, Q)
$$

where the sum is over pairs $(P, Q)$, where $P$ and $Q$ are of the form $2 \cdot \xi^{k}$ for $k=0,1,2, \ldots$ and satisfy the inequalities

$$
T\left(\gamma_{0}\right) \leq P Q \leq x \quad \text { and } \quad P \leq \xi Q .
$$

Finally note that (12) implies that we can even restrict the summation to the cases

$$
4(P Q)^{\frac{1}{2}+\gamma} \geq Q
$$

since otherwise $R_{\gamma}(P, Q)=0$. Combining (19) and (20), we deduce that $P$ and $Q$ satisfy the inequalities

$$
P \leq \xi Q \quad \text { and } \quad \kappa_{0} Q^{\frac{1-2 \gamma}{1+2 \gamma}} \leq P \leq x Q^{-1} \quad \text { with } \kappa_{0}=4^{-\frac{2}{1+2 \gamma}} .
$$


The inequality (18) can be easily transformed into a lower bound on $H_{\gamma}^{0}(x)$ if one replaces $\Theta_{0}$ by $\Theta_{0}^{\prime}$ with $\Theta_{0}^{\prime}:=\theta_{0}\left(\xi^{2} P Q\right)$ in the definition (16) of $R_{\gamma}(P, Q)$. We note that

$$
\Theta_{0}^{\prime}-\Theta_{0}=O\left(\Theta_{0} \mathscr{L}^{-1}\right),
$$

as a result of Lemma 8 and the fineness of the cutting of the sum $H_{\gamma}^{0}(x)$ (see (18)).

\section{Proof of Theorem 1}

The first purpose of this section is to prove the following:

Proposition 1. Let $\gamma_{0}>0$. Then uniformly for $\gamma$ satisfying

$$
\frac{12}{25}+\gamma_{0} \leq \gamma \leq \frac{1}{2}-\gamma_{0}
$$

and for $(P, Q)$ satisfying the conditions (21), one has the equality

$$
R_{\gamma}(P, Q)=\frac{1}{2}(P Q)^{\gamma-\frac{1}{2}}\left(1+O\left(\mathscr{L}^{-1}\right)\right)\left(\sum_{\substack{p \approx P \\ p q \leq x}} \sum_{\substack{q \approx Q \\ p<q}} 1\right)+O\left(x^{\frac{1}{2}+\gamma} \mathscr{L}^{-6}\right)+O\left(Q \mathscr{L}^{-4}\right) \text {. }
$$

Our proof depends on the size of $P$ compared with $Q$.

4.1. When $\boldsymbol{P}$ is small. Let $\mathscr{E}\left(p, \Theta_{0}\right)$ denote the set of congruence classes $s$ mod $p$ such that $0<\bar{s} / p \leq \Theta_{0}$. Of course, $\bar{s}$ is the multiplicative inverse of $s \bmod p$. By the definition (17) and by Lemma 8 , its cardinality satisfies

$$
\# \mathscr{E}\left(p, \Theta_{0}\right)=\left(\frac{1}{2}+O\left(\mathscr{L}^{-1}\right)\right) P^{\frac{1}{2}+\gamma} Q^{\gamma-\frac{1}{2}}+O(1) .
$$

Let

$$
y_{p}:=\max (Q, p) \quad \text { and } \quad z_{p}:=\min (\xi Q, x / p) .
$$

With this definition, we have the equality

$$
R_{\gamma}(P, Q)=\sum_{\substack{p \approx P \\ y_{p} \leq z_{p}}} \sum_{s \in \mathscr{E}\left(p, \Theta_{0}\right)}\left(\pi\left(z_{p} ; p, s\right)-\pi\left(y_{p} ; p, s\right)\right) .
$$

For $(P, Q)$ satisfying $(21)$, the trivial estimate

$$
\left(\pi\left(z_{p} ; p, s\right)-\pi\left(y_{p} ; p, s\right)\right) \leq Q / p+1 \ll Q / p
$$

inserted in (26) gives the bound

$$
R_{\gamma}(P, Q) \ll(P Q)^{\frac{1}{2}+\gamma}+Q \ll(P Q)^{\frac{1}{2}+\gamma}
$$

by (20). Hence, for the proof of Proposition 1, we may add the extra condition

$$
P Q \geq x \mathscr{L}^{-12} \text {. }
$$


The equalities (24) and (26) and Lemma 5 allow us to improve (27) by

$$
\begin{aligned}
R_{\gamma}(P, Q)=\left[\left(\frac{1}{2}+O\left(\mathscr{L}^{-1}\right)\right) P^{\frac{1}{2}+\gamma} Q^{\gamma-\frac{1}{2}}\right. & +O(1)]\left(\sum_{\substack{p \approx P \\
p q \leq x}} \sum_{\substack{q \approx Q \\
p<q}} \frac{1}{\varphi(p)}\right) \\
& +O\left((P Q)^{\frac{1}{2}+\gamma} \mathscr{L}^{-6}\right)+O\left(Q \mathscr{L}^{-6}\right)
\end{aligned}
$$

provided

$$
P \leq Q^{\frac{1}{2}} \mathscr{L}^{-100} .
$$

The contribution of the $O(1)$-term to the right-hand side of (29) is bounded by $Q \mathscr{L}^{-4}$, up to a multiplicative constant. Recalling the restriction (28), we see that the proof of Proposition 1 is complete in the particular case

$$
P \leq x^{\frac{1}{3}} \mathscr{L}^{-100} .
$$

4.2. Medium values of $\boldsymbol{P}$. We apply Lemma 1 with the choices

$$
\beta=\Theta_{0}, \quad \Delta=\Theta_{0} \mathscr{L}^{-3}, \quad r=4 .
$$

We then have the inequalities

$$
\sum_{\substack{p \approx P \\ p q \leq x}} \sum_{\substack{q \approx Q \\ p<q}} \psi^{-}\left(\frac{\bar{q}}{p}\right) \leq R_{\gamma}(P, Q) \leq \sum_{\substack{p \approx P \\ p q \leq x}} \sum_{\substack{q \approx Q \\ p<q}} \psi^{+}\left(\frac{\bar{q}}{p}\right) .
$$

We only study the upper bound of $R_{\gamma}(P, Q)$ in (32). We recall the definitions (9) and (25). We apply Lemma 1 (in a slightly weaker form) and decompose the sums according to the values of $m$ and whether $p$ and $m$ are coprime. This gives

$$
\begin{aligned}
& \sum_{\substack{p \approx P \\
p q \leq x}} \sum_{\substack{q \approx Q \\
p<\bar{q}}} \psi^{+}\left(\frac{\bar{q}}{p}\right) \\
& \leq(\beta+\Delta) \sum_{\substack{p \approx P \\
p q \leq x}} \sum_{\substack{q \approx Q \\
p<\bar{q}}} \\
& +2 \sum_{p \approx P}\left\{\sum_{\substack{1 \leq|m| \leq \Delta^{-1} \\
p \nmid m}} \frac{1}{\pi|m|}+\sum_{|m|>\Delta^{-1}} \frac{256}{\pi^{5}|m|^{5} \Delta^{4}}\right. \\
& \left.\quad+\sum_{\substack{1 \leq|m| \leq \Delta^{-1} \\
p \mid m}} \frac{2}{\pi|m|}+\sum_{\substack{|m|>\Delta^{-1} \\
p \mid m}} \frac{256}{\pi^{5}|m|^{5} \Delta^{4}}\right\}\left|S_{p}\left(m ; y_{p}, z_{p}\right)\right| .
\end{aligned}
$$

It remains to apply Lemma 2 when $p \nmid m$, or the trivial inequality $\left|S_{p}\right| \leq Q$ otherwise, and to sum over $m$ to obtain the inequality 


$$
\begin{aligned}
\sum_{\substack{p \approx P \\
p q \leq x}} \sum_{\substack{q \approx Q \\
p<q}} \psi^{+}\left(\frac{\bar{q}}{p}\right) \leq(\beta+\Delta) \sum_{\substack{p \approx P \\
p q \leq x}} \sum_{\substack{q \approx Q \\
p<q}} 1 \\
\quad+O\left(\sum_{p \approx P}\left\{(\mathscr{L}+1)\left(p^{-\frac{1}{2}} Q+p^{\frac{1}{4}} Q^{\frac{4}{5}}\right) \mathscr{L}^{2}+\left(p^{-1} \mathscr{L}+p^{-1}\right) Q\right\}\right) .
\end{aligned}
$$

Using the upper bound $\sum_{p \approx P} 1 \ll_{\gamma_{0}} P \mathscr{L}^{-2}$, we see that the error term satisfies

$$
\text { error term } \ll_{\gamma_{0}}\left(P^{\frac{1}{2}} Q+P^{\frac{5}{4}} Q^{\frac{4}{5}}\right) \mathscr{L} \text {. }
$$

By Lemma 8 and (28), we have the equality

$$
\Theta_{0}=\frac{1}{2}(P Q)^{\gamma-\frac{1}{2}}+O\left((P Q)^{2 \gamma-1}\right)=\frac{1}{2}(P Q)^{\gamma-\frac{1}{2}}\left(1+O\left(\mathscr{L}^{-3}\right)\right),
$$

which, combined with (32), (34) and (35) gives the inequality

$$
\begin{aligned}
& R_{\gamma}(P, Q) \\
& \quad=\frac{1}{2}(P Q)^{\gamma-\frac{1}{2}}\left(1+O\left(\mathscr{L}^{-3}\right)\right)\left(\sum_{\substack{p \approx P \\
p q \leq x}} \sum_{\substack{q \approx Q \\
p<q}} 1\right)+O\left(\left(P^{\frac{1}{2}} Q+P^{\frac{5}{4}} Q^{\frac{4}{5}}\right) \mathscr{L}\right) .
\end{aligned}
$$

Recalling the restrictions (21), we see that (36) implies Proposition 1 as soon as $P$ satisfies the inequalities

$$
P \geq x^{1-2 \gamma} \mathscr{L}^{14} \text { and } P \leq x^{\frac{20}{9} \gamma-\frac{2}{3}} \mathscr{L}^{-16}
$$

4.3. Large values of $\boldsymbol{P}$. Actually, in (33) we may benefit from the summation over $p \approx P$ by appealing to Lemma 3 instead of Lemma 2 . By the same technique as in Section 4.2 , we arrive at the equality

$$
R_{\gamma}(P, Q)=\Theta_{0}\left(1+O\left(\mathscr{L}^{-3}\right)\right)\left(\sum_{\substack{p \approx P \\ p q \leq x}} \sum_{\substack{q \approx Q \\ p<q}} 1\right)+O_{\epsilon}\left(\left(P^{\frac{13}{10}} Q^{\frac{3}{5}}+P^{\frac{13}{12}} Q^{\frac{5}{6}}\right) x^{\epsilon}\right)
$$

provided $P^{\frac{3}{2}} \geq Q$ and $\epsilon$ is an arbitrary positive number. Hence, by (21) and (28), we see that (38) implies Proposition 1 as soon as $P$ satisfies the extra conditions

$$
P \geq x^{\frac{2}{5}}, \quad P \leq x^{\frac{10}{7} \gamma-\frac{1}{7}-2 \epsilon} \quad \text { and } \quad P \leq x^{4 \gamma-\frac{4}{3}-5 \epsilon} .
$$

Suppose now that $\gamma$ satisfies (23) and that $P$ satisfies $1 \leq P \leq 2 \sqrt{x}$. Then we see that $P$ satisfies at least one of the sets of conditions (31), (37) or (39). This completes the proof of Proposition 1. 
4.4. Conclusion of the proof of Theorem 1. We insert the expansion of $R_{\gamma}(P, Q)$ given in Proposition 1 in the right-hand side of (18) and sum over $(P, Q)$ satisfying (21). Recall that the numbers $P$ and $Q$ are of the shape $2 \cdot \xi^{k}$. We first consider the contribution of the term $O\left(Q \mathscr{L}^{-4}\right)$. By (21), this contribution satisfies

$$
\begin{aligned}
O\left(Q \mathscr{L}^{-4}\right) \text { term } & \ll \mathscr{L}^{-4} \sum_{Q} Q \sum_{\substack{\kappa_{0} Q^{\frac{1-2 \gamma}{1+2 \gamma}} \leq P<x Q^{-1}\\
}} 1 \\
& \ll \mathscr{L}^{-3} \sum_{Q \leq\left(\frac{x}{\kappa_{0}}\right)^{\frac{1}{2}+\gamma}} Q\left(\log \left(\frac{x}{\kappa_{0}} Q^{-\frac{2}{1+2 \gamma}}\right)+1\right) \\
& \ll \mathscr{L}^{-3}\left\{\sum_{Q \leq\left(\frac{x}{\kappa_{0}}\right)^{\frac{1}{2}+\gamma} \mathscr{L}^{-1}} Q \mathscr{L}+\log \mathscr{L} \sum_{\left(\frac{x}{\kappa_{0}}\right)^{\frac{1}{2}+\gamma} \mathscr{L}^{-1} \leq Q \leq\left(\frac{x}{\kappa_{0}}\right)^{\frac{1}{2}+\gamma}} Q\right\} \\
& \ll x^{\frac{1}{2}+\gamma \mathscr{L}^{-\frac{3}{2}} .}
\end{aligned}
$$

Since the number of $(P, Q)$ satisfying (21) is $O\left(\mathscr{L}^{4}\right)$, the contribution of the term $O\left(x^{\frac{1}{2}+\gamma} \mathscr{L}^{-6}\right)$ (coming from Proposition 1) is $O\left(x^{\frac{1}{2}+\gamma} \mathscr{L}^{-2}\right)$. From the above considerations, we deduce the inequality

$$
H_{\gamma}^{0}(x) \leq\left(\frac{1}{2}+o(1)\right) \sum_{P} \sum_{Q}(P Q)^{\gamma-\frac{1}{2}}\left(\sum_{\substack{p \approx P \\ p q \leq x}} \sum_{\substack{q \approx Q \\ p<q}} 1\right)+O\left(x^{\frac{1}{2}+\gamma} \mathscr{L}^{-2}\right),
$$

where $P$ and $Q$ satisfy (21). We now want to drop the dissection parameters $P$ and $Q$. To do so, we remark that $(P Q)^{\gamma-\frac{1}{2}}=(1+o(1))(p q)^{\gamma-\frac{1}{2}}$ for $p \approx P$ and $q \approx Q$. We gather the rectangles of summation $] P, \xi P] \times] Q, \xi Q]$ to deduce the inequality

$$
H_{\gamma}^{0}(x) \leq\left(\frac{1}{2}+o(1)\right)\left(\sum_{p<q \leq x / p} \sum_{p q)^{\gamma-\frac{1}{2}}}\right)+O\left(x^{\frac{1}{2}+\gamma} \mathscr{L}^{-2}\right) .
$$

By the prime number theorem, we have

$$
\sum_{p<q \leq x / p} \sum_{p}(p q)^{\gamma-\frac{1}{2}} \sim \int_{x^{\frac{1}{2}}}^{x^{\frac{1}{2}+\gamma}} \frac{y^{\gamma-\frac{1}{2}}}{\log y} d y \int_{3}^{x y^{-1}} \frac{z^{\gamma-\frac{1}{2}}}{\log z} d z \quad(x \rightarrow \infty) .
$$

Write $y:=x^{u}$ and $z:=x^{v}$ to deduce

$$
\begin{aligned}
\sum_{p<q \leq x / p}(p q)^{\gamma-\frac{1}{2}} & \sim \int_{\frac{1}{2}}^{\frac{1}{2}+\gamma} \frac{x^{u\left(\gamma+\frac{1}{2}\right)}}{u} d u \int_{\frac{\log 3}{\log x}}^{1-u} \frac{x^{v\left(\gamma+\frac{1}{2}\right)}}{v} d v \\
& \sim \int_{\frac{1}{2}}^{\frac{1}{2}+\gamma} \frac{\left.x^{u\left(\gamma+\frac{1}{2}\right.}\right)}{u} \cdot \frac{x^{(1-u)\left(\gamma+\frac{1}{2}\right)}}{(1-u)\left(\gamma+\frac{1}{2}\right) \log x} d u \sim C(\gamma) \frac{x^{\frac{1}{2}+\gamma}}{\log x},
\end{aligned}
$$


where $C(\gamma)$ is defined in (5).

The study of $H_{\gamma}^{1}(x)$ defined in (15) is similar to the study of $H_{\gamma}^{0}(x)$. Combining (15), (40) and (41), we finally arrive at the inequality

$$
H_{\gamma}(x) \leq(1+o(1)) C(\gamma) \frac{x^{\frac{1}{2}+\gamma}}{\log 2 x} .
$$

To produce a lower bound for $H_{\gamma}^{0}(x)$, we follow the idea presented at the end of Section 3.2, which consists of replacing the constant $\Theta_{0}$ by $\Theta_{0}^{\prime}$ in the definition of $R_{\gamma}(P, Q)$. By (22), we also obtain the inequalities

$$
H_{\gamma}^{0}(x), H_{\gamma}^{1}(x) \geq(1-o(1)) \frac{C(\gamma)}{2} \cdot \frac{x^{\frac{1}{2}+\gamma}}{\log 2 x}
$$

as $x$ tends to infinity. Summing these two inequalities, we arrive at

$$
H_{\gamma}(x) \geq(1-o(1)) C(\gamma) \frac{x^{\frac{1}{2}+\gamma}}{\log 2 x} .
$$

Combining with (42), this completes the proof of Theorem 1.

\section{Proof of Theorem 2}

We still suppose that (13) is satisfied and that $P Q$ is large enough, which means $P Q \geq T\left(\gamma_{0}\right)$, where $T\left(\gamma_{0}\right)$ is defined in Lemma 8. Since we are searching for an upper bound, it is useless to work with a very thin cutting up as in (16). So let

$$
\begin{aligned}
& S_{\gamma}^{0}(P, Q):=\#\left\{(p, q): p \sim P, q \sim Q, p<q, 0<\frac{\bar{q}}{p} \leq \Theta_{0}\right\}, \\
& S_{\gamma}^{1}(P, Q):=\#\left\{(p, q): p \sim P, q \sim Q, p<q, 1-\Theta_{1}<\frac{\bar{q}}{p}<1\right\},
\end{aligned}
$$

where $\Theta_{0}$ is still defined by $(17)$ and $\Theta_{1}=\theta_{1}(P Q)$. We then have the inequality

$$
H_{\gamma}(x) \leq \sum_{(P, Q)} \sum_{\gamma} S_{\gamma}^{0}(P, Q)+\sum_{(P, Q)} \sum_{\gamma} S_{\gamma}^{1}(P, Q)+O\left(T\left(\gamma_{0}\right)\right),
$$

where $P$ and $Q$ are powers of 2 and satisfy $P \leq 2 Q$ and $T\left(\gamma_{0}\right) \leq P Q \leq x$. We will focus our study on the case of $S_{\gamma}^{0}(P, Q)$.

Define

$$
L:=P^{\gamma+\frac{1}{2}} Q^{\gamma-\frac{1}{2}}
$$

If $(p, q)$ contributes to $S_{\gamma}^{0}(P, Q)$, then we have the equality (4) for some $\ell$ satisfying $1 \leq \ell \ll L$. Hence, we have the inequality

where

$$
S_{\gamma}^{0}(P, Q) \leq \sum_{1 \leq \ell \ll L} \sum_{n \asymp \ell Q / P} F(\ell, n, P, Q),
$$


- the constants implicit in the symbols $\ll$ and $\asymp$ depend on $\gamma_{0}$ only and

- $F(\ell, n, P, Q)$ is the number of solutions of the equation $\ell q-n p=1$ in primes $p \sim P$ and $q \sim Q$.

By Lemma 4, we have the inequality

$$
F(\ell, n, P, Q) \ll \frac{\ell Q}{\varphi(\ell n)} \cdot \log ^{-2} z
$$

provided $z \leq P^{\frac{1}{2}}$ and $\ell Q \geq \ell n z \log ^{4} z$. By the order of magnitude of the parameters, this last condition reduces to

$$
P \gg \ell z \log ^{4} z
$$

However, since we have $\ell \ll L$, this inequality is satisfied as soon as

$$
(P Q)^{\frac{1}{2}-\gamma} \gg z^{2} \text {. }
$$

Choose $z:=(P Q)^{\frac{1}{6}-\frac{\gamma}{3}}$. With this choice of $z$ inserted in (48) and by (47), we obtain the inequality

$$
S_{\gamma}^{0}(P, Q) \ll_{\gamma_{0}} \frac{Q}{\log ^{2}(P Q)} \sum_{1 \leq \ell \ll L} \ell \sum_{n \asymp \ell Q / P} \frac{1}{\varphi(\ell n)} .
$$

Recall the inequality $\varphi(\ell n) \geq \varphi(\ell) \varphi(n)$ and the bound $\sum_{t \sim T} \varphi^{-1}(t) \ll 1$, which is uniform in $T \geq 1$. Then summing over $\ell$ and $n$ in (49), we deduce the inequality

$$
S_{\gamma}^{0}(P, Q) \ll_{\gamma_{0}} L Q \log ^{-2}(P Q) \ll_{\gamma_{0}}(P Q)^{\gamma+\frac{1}{2}} \log ^{-2}(P Q) .
$$

This bound also holds for $S_{\gamma}^{1}(P, Q)$. Inserting this bound in (45) and summing over $(P, Q)$ such that $P Q \leq x$, we conclude the proof of Theorem 2 .

\section{Proof of Theorem 3}

We now suppose that

$$
\frac{15}{98}+\gamma_{0} \leq \gamma \leq \frac{13}{27}
$$

since the case where $\gamma$ takes large values is covered by Theorem 1. Define also

$$
K_{0}:=\frac{17-49 \gamma_{0}}{32-4 \gamma_{0}} \quad\left(<\frac{17}{32}\right) .
$$

To deal with the lower bound of $H_{\gamma}(x)$, we consider

$$
T_{\gamma}^{0}(P, Q):=\#\left\{(p, q): p \sim P, q \sim Q, 0<\frac{\bar{q}}{p} \leq \Theta_{0}^{\dagger}\right\}
$$

with

$$
\Theta_{0}^{\dagger}:=\theta_{0}(4 P Q),
$$


where $\theta_{0}$ is defined in Lemma 8 . We have the inequality

$$
H_{\gamma}(x) \geq H_{\gamma}^{0}(x) \geq \sum_{P} \sum_{Q} T_{\gamma}^{0}(P, Q),
$$

where $H_{\gamma}^{0}(x)$ is defined in (15) and the sum is over the pairs $(P, Q)$ of the form $\left(2^{k}, 2^{\ell}\right)$ with

$$
P \leq Q^{K_{0}}, \quad x / 16 \leq P Q \leq x / 4, \quad P \leq Q / 2 \quad \text { and } \quad 1 \leq L \leq Q^{\beta_{K_{0}}},
$$

where $L$ is defined in (46) and $\beta_{K}$ is the constant introduced in Lemma 7. If the triple $(\ell, p, q)$ is such that $1 \leq \ell \ll L, p \sim P$ and $q \sim Q$ and satisfies $\ell q-n p=1$ for some integer $n$, then it contributes to $T_{\gamma}^{0}(P, Q)$. This leads to the inequality

$$
T_{\gamma}^{0}(P, Q) \geq \sum_{p \sim P} \sum_{1 \leq \ell \ll L}(\pi(2 Q ; p, \bar{\ell})-\pi(Q ; p, \bar{\ell})) .
$$

Thanks to (52), we can apply Lemma 7, giving

$$
T_{\gamma}^{0}(P, Q) \geq \alpha_{K_{0}} \sum_{p \sim P} L \cdot \frac{Q}{\varphi(p) \log 2 Q}-O\left(\frac{P}{\log ^{2} 2 P} \cdot L \cdot \frac{Q}{P \log 2 Q}\right),
$$

which simplifies into

$$
T_{\gamma}^{0}(P, Q) \geq \frac{\alpha_{K_{0}}}{2} \cdot \frac{L Q}{\log 2 P \log 2 Q}
$$

for $x \geq x_{0}$ and $(P, Q)$ satisfying (52).

In terms of $P$, the conditions (52) and $L \gg 1$ reduce to

$$
P \ll x^{\frac{K_{0}}{1+K_{0}}} \quad \text { and } \quad x^{\frac{1}{2}-\gamma} \ll P \ll x^{\left(\frac{1}{2}+\beta_{K_{0}}-\gamma\right) /\left(1+\beta_{K_{0}}\right)} .
$$

The definition of $K_{0}$ implies the inequality

$$
\frac{K_{0}}{1+K_{0}}-\left(\frac{1}{2}-\gamma\right) \geq \frac{K_{0}}{1+K_{0}}-\left(\frac{17}{49}-\gamma_{0}\right) \gg_{\gamma_{0}} 1 \text {. }
$$

Combining with the inequality $\beta_{K_{0}}>0$, we see that there are $\gg_{\gamma_{0}} \mathscr{L}$ values of $P$ of the form $P=2^{k}$ satisfying (54). Since we also have $x /(16 P) \leq Q \leq x /(4 P)$, we deduce that there are $\gg_{\gamma_{0}} \mathscr{L}$ pairs $(P, Q)$ satisfying (52). It remains to insert the lower bound (53) in (51) and to sum over the suitable $(P, Q)$ to deduce

$$
H_{\gamma}^{0}(x) \gg_{\gamma_{0}} x^{\frac{1}{2}+\gamma} \mathscr{L}^{-1}
$$

This completes the proof of Theorem 3 .

Remark. Not using Lemma 7 but only Lemma 5, one proves Theorem 3 but under the more restrictive condition $\frac{1}{6}+\gamma_{0} \leq \gamma \leq 1-\gamma_{0}$. 


\section{A conjectural formula}

One may conjecture that for every $\gamma_{0}>0$, one has

$$
H_{\gamma}(x) \sim C(\gamma) \frac{x^{\frac{1}{2}+\gamma}}{\log x}
$$

as $x \rightarrow \infty$ uniformly under the condition (13). This conjecture, if true, would be an important extension of Theorem 1. However, (55) is a consequence of the Elliott-Halberstam Conjecture (see [Friedlander and Iwaniec 2010, page 406] for instance).

Conjecture 1. For any $\epsilon>0$ and any $A>0$, one has

$$
\sum_{r \leq x^{1-\epsilon}} \max _{(s, r)=1}\left|\pi(x ; r, s)-\frac{\pi(x)}{\varphi(r)}\right|=O_{\epsilon, A}\left(x \mathscr{L}^{-A}\right) .
$$

This conjecture can be interpreted as a considerable improvement of Lemma 5 since it gives the average behavior of the function $\pi(x ; r, s)$ for almost all $r \leq x^{1-\epsilon}$.

We now give some indications on how to deduce (55) from Conjecture 1. First of all, one applies the formula (56) to evaluate $R_{\gamma}(P, Q)$ as written in (26). This shows that (29) is true uniformly for $P \leq Q x^{-\epsilon}$ (compare with (30)). Summing over all these $(P, Q)$, we see that their contribution to $H_{\gamma}(x)$ is $\sim(C(\gamma)-O(\epsilon)) x^{\frac{1}{2}+\gamma} \mathscr{L}^{-1}$ by a computation analogous to (41) and (42) with uniformity given by (13).

For the remaining $(P, Q)$ (those that satisfy $\left.Q x^{-\epsilon} \leq P \leq \xi \cdot Q\right)$, we apply the two-dimensional sieve as in Section 5. Then one shows that their contribution to $H_{\gamma}(x)$ is $O_{\gamma_{0}}\left(\epsilon x^{\frac{1}{2}+\gamma} \mathscr{L}^{-1}\right)$. Summing up these two contributions and letting $\epsilon$ tend to 0 , we get (55).

\section{Acknowledgements}

The author warmly thanks I. Shparlinski for indicating the work of B. Bzdęga and for generously sharing his ideas about this problem. We also thank F. Jouve for his comments on a preliminary version of that work and the referee for his judicious remarks, which clarified some parts of the present paper.

\section{References}

[Baker and Harman 1996] R. C. Baker and G. Harman, "The Brun-Titchmarsh theorem on average", pp. 39-103 in Analytic number theory, Vol. 1 (Allerton Park, IL, 1995), edited by B. C. Berndt et al., Progr. Math. 138, Birkhäuser, Boston, MA, 1996. MR 97h:11096 Zbl 0853.11078

[Bombieri et al. 1987] E. Bombieri, J. B. Friedlander, and H. Iwaniec, "Primes in arithmetic progressions to large moduli, II”, Math. Ann. 277:3 (1987), 361-393. MR 88f:11085 Zbl 0625.10036

[Bombieri et al. 1989] E. Bombieri, J. B. Friedlander, and H. Iwaniec, "Primes in arithmetic progressions to large moduli, III”, J. Amer. Math. Soc. 2:2 (1989), 215-224. MR 89m:11087 Zbl 0674.10036 
[Bzdęga 2012] B. Bzdęga, "Sparse binary cyclotomic polynomials", J. Number Theory 132:3 (2012), 410-413. MR 2875347 Zbl pre06005607

[Carlitz 1966] L. Carlitz, "The number of terms in the cyclotomic polynomial $F_{p q}(x)$ ", Amer. Math. Monthly 73 (1966), 979-981. MR 34 \#2517 Zbl 0146.26704

[Fouvry 1985] É. Fouvry, "Théorème de Brun-Titchmarsh: application au théorème de Fermat", Invent. Math. 79:2 (1985), 383-407. MR 86g:11052 Zbl 0557.10035

[Fouvry and Michel 1998] É. Fouvry and P. Michel, "Sur certaines sommes d'exponentielles sur les nombres premiers", Ann. Sci. École Norm. Sup. (4) 31:1 (1998), 93-130. MR 98m:11088 Zbl 0915.11045

[Fouvry and Shparlinski 2011] É. Fouvry and I. E. Shparlinski, "On a ternary quadratic form over primes", Acta Arith. 150:3 (2011), 285-314. MR 2842959 Zbl 1243.11093

[Friedlander and Iwaniec 2010] J. Friedlander and H. Iwaniec, Opera de cribro, American Mathematical Society Colloquium Publications 57, American Mathematical Society, Providence, RI, 2010. MR 2011d:11227 Zbl 1226.11099

[Garaev 2010] M. Z. Garaev, "An estimate for Kloosterman sums with primes and its application”, Mat. Zametki 88:3 (2010), 365-373. In Russian; translated in Math. Notes 88:3 (2010), 330-337. MR 2882176 Zbl pre05980675

[Habsieger and Sivak-Fischler 2010] L. Habsieger and J. Sivak-Fischler, "An effective version of the Bombieri-Vinogradov theorem, and applications to Chen's theorem and to sums of primes and powers of two", Arch. Math. (Basel) 95:6 (2010), 557-566. MR 2011m:11190 Zbl 05833792

[Hildebrand 1985] A. Hildebrand, "On a conjecture of Balog", Proc. Amer. Math. Soc. 95:4 (1985), 517-523. MR 87c:11001 Zbl 0597.10056

[Iwaniec and Kowalski 2004] H. Iwaniec and E. Kowalski, Analytic number theory, American Mathematical Society Colloquium Publications 53, American Mathematical Society, Providence, RI, 2004. MR 2005h:11005 Zbl 1059.11001

[Lam and Leung 1996] T. Y. Lam and K. H. Leung, "On the cyclotomic polynomial $\Phi_{p q}(X)$ ", Amer. Math. Monthly 103:7 (1996), 562-564. MR 97h:11150 Zbl 0868.11016

[Mikawa 2001] H. Mikawa, "On primes in arithmetic progressions", Tsukuba J. Math. 25:1 (2001), 121-153. MR 2002c:11116 Zbl 1017.11049

[Rousselet 1988] B. Rousselet, "Inégalités de type Brun-Titchmarsh en moyenne", pp. 91-123 in Groupe de travail en théorie analytique et élémentaire des nombres, 1986-1987, Publ. Math. Orsay 88, Univ. Paris XI, Orsay, 1988. MR 89g:11076 Zbl 0669.10067

[Vinogradov 1954] I. M. Vinogradov, The method of trigonometrical sums in the theory of numbers, Interscience Publishers, New York, 1954. Zbl 0055.27504

Communicated by Philippe Michel

Received 2012-02-20 Revised 2012-06-29 Accepted 2012-08-13

etienne.fouvry@math.u-psud.fr Laboratoire de Mathématique,

Campus d'Orsay, Université de Paris-Sud, Bâtiment 425 UMR 8628, 91405 Orsay Cedex, France http://www.math.u-psud.fr/ fouvry/ 


\section{Algebra \& Number Theory}

msp.org/ant

\section{EDITORS}

MANAGING EDITOR

Bjorn Poonen

Massachusetts Institute of Technology

Cambridge, USA

\author{
EDITORIAL BOARD CHAIR \\ David Eisenbud \\ University of California \\ Berkeley, USA
}

\section{BOARD OF EDITORS}

Georgia Benkart

Dave Benson

Richard E. Borcherds

John H. Coates

J-L. Colliot-Thélène

Brian D. Conrad

Hélène Esnault

Hubert Flenner

Edward Frenkel

Andrew Granville

Joseph Gubeladze

Ehud Hrushovski

Craig Huneke

Mikhail Kapranov

Yujiro Kawamata

János Kollár

Yuri Manin

Barry Mazur

Philippe Michel
University of Wisconsin, Madison, USA

University of Aberdeen, Scotland

University of California, Berkeley, USA

University of Cambridge, UK

CNRS, Université Paris-Sud, France

University of Michigan, USA

Freie Universität Berlin, Germany

Ruhr-Universität, Germany

University of California, Berkeley, USA

Université de Montréal, Canada

San Francisco State University, USA

Hebrew University, Israel

University of Virginia, USA

Yale University, USA

University of Tokyo, Japan

Princeton University, USA

Northwestern University, USA

Harvard University, USA

École Polytechnique Fédérale de Lausanne
Susan Montgomery

Shigefumi Mori

Raman Parimala

Jonathan Pila

Victor Reiner

Karl Rubin

Peter Sarnak

Joseph H. Silverman

Michael Singer

Vasudevan Srinivas

J. Toby Stafford

Bernd Sturmfels

Richard Taylor

Ravi Vakil

Michel van den Bergh

Marie-France Vignéras

Kei-Ichi Watanabe

Efim Zelmanov
University of Southern California, USA

RIMS, Kyoto University, Japan

Emory University, USA

University of Oxford, UK

University of Minnesota, USA

University of California, Irvine, USA

Princeton University, USA

Brown University, USA

North Carolina State University, USA

Tata Inst. of Fund. Research, India

University of Michigan, USA

University of California, Berkeley, USA

Harvard University, USA

Stanford University, USA

Hasselt University, Belgium

Université Paris VII, France

Nihon University, Japan

University of California, San Diego, USA

\section{PRODUCTION}

production@msp.org

Silvio Levy, Scientific Editor

See inside back cover or msp.org/ant for submission instructions.

The subscription price for 2013 is US \$200/year for the electronic version, and \$350/year ( $\$ 40$, if shipping outside the US) for print and electronic. Subscriptions, requests for back issues and changes of subscribers address should be sent to MSP.

Algebra \& Number Theory (ISSN 1944-7833 electronic, 1937-0652 printed) at Mathematical Sciences Publishers, 798 Evans Hall \#3840, c/o University of California, Berkeley, CA 94720-3840 is published continuously online. Periodical rate postage paid at Berkeley, CA 94704, and additional mailing offices.

ANT peer review and production are managed by EditFLOW ${ }^{\circledR}$ from Mathematical Sciences Publishers.

\section{PUBLISHED BY}

- mathematical sciences publishers

nonprofit scientific publishing

http://msp.org/

(C) 2013 Mathematical Sciences Publishers 


\section{Algebra \& Number Theory}

Volume $7 \quad$ No. $5 \quad 2013$

Sharp upper bounds for the Betti numbers of a given Hilbert polynomial

GiUlio CAVIGLia and SATOShi Murai

Comparing numerical dimensions

1065

BRIAN LEHMANN

Some consequences of a formula of Mazur and Rubin for arithmetic local constants JAN NEKOVÁŘ

Quantized mixed tensor space and Schur-Weyl duality

RICHARD DIPPER, STEPHEN DOTY and FRIEDERIKE STOLL

Weakly commensurable $S$-arithmetic subgroups in almost simple algebraic groups of

types B and C

SKIP GARIBALDI and ANDREI RAPINCHUK

Minimisation and reduction of 5-coverings of elliptic curves

1179

TOM FISHER

On binary cyclotomic polynomials

1207

ÉTIENNE FOUVRY

Local and global canonical height functions for affine space regular automorphisms

SHU KAWAGUCHI

On the ranks of the 2-Selmer groups of twists of a given elliptic curve

1101

1121

147

DANIEL M. KANE 